・方法・

\title{
7个林木大小多样性指数表达能力比较
}

\author{
娄明华 ${ }^{1}$ 白 超 ${ }^{*}$ 惠刚盈 ${ }^{3}$ 汤孟平 ${ }^{4}$ \\ 1 (宁波市农业科学研究院, 浙江宁波 315040) \\ 2 (宁波市环境保护科学研究设计院, 浙江宁波 315010) \\ 3 (中国林业科学研究院林业研究所, 国家林业局林木培育重点实验室, 北京 100091) \\ 4 (浙江农林大学森林生态系统碳循环与固碳减排省重点实验室, 杭州 311300)
}

摘要：林木大小多样性直接反映森林生态系统的健康与稳定, 客观恰当地表达大小多样性对于评价天然林或人工 林的经济、生态、社会价值及其经营效果至关重要。本研究选用7个林木大小多样性指数，其中 4 个与距离无关 (Simpson大小多样性指数 $D_{N} 、$ Shannon大小多样性指数 $H_{N} 、$ 断面积Gini系数 $G C$ 和直径变异系数 $C V_{d}$ ), 3 个与距离有 关(Simpson大小分化度指数 $D_{T} 、$ Shannon大小分化度指数 $H_{T}$ 和大小分化度均值指数 $\bar{T}$ ), 通过 6 组模拟林分和 4 块实 测林分比较分析了它们的表达能力。结果表明: 不考虑极端情况(极端情况为对比林分林木大小混交不同但林木直 径构成完全相同), $G C 、 C V_{d} 、 \bar{T} 、 D_{T}$ 和 $H_{T}$ 能客观恰当地表达不同径级分布林分的林木大小多样性差异, 其中 $C V_{d}$ 区分能力最强, $G C$ 次之。若考虑极端情况, 只有 $\bar{T} 、 D_{T}$ 和 $H_{T}$ 能区分出不同大小混交程度林分的林木大小多样性差 异。本研究认为 $C V_{d}$ 和 $G C$ 因计算简单，易于实际应用，在营林活动中可作为分析林木大小多样性的首选指数; $\bar{T}$ 因能 识别不同大小混交程度林分的空间差异，即对林分更新变化敏感，适用于动态分析林分的结构特征。

关键词：林木大小多样性; 直径分布; 大小分化度; 大小混交; 大小隔离

\section{Comparison of distinguish ability on seven tree size diversity indices}

\author{
Minghua $\mathrm{Lou}^{1}$, Chao Bai ${ }^{2 *}$, Gangying $\mathrm{Hui}^{3}$, Mengping Tang ${ }^{4}$ \\ 1 Ningbo Academy of Agricultural Sciences, Ningbo, Zhejiang 315040 \\ 2 Ningbo Scientific Research and Design Institute of Environmental Protection, Ningbo, Zhejiang 315010 \\ 3 Key Laboratory of Tree Breeding and Cultivation of State Forestry Administration, Research Institute of Forestry, \\ Chinese Academy of Forestry, Beijing 100091 \\ 4 Zhejiang Provincial Key Laboratory of Carbon Cycling in Forest Ecosystems and Carbon Sequestration, Zhejiang A \& \\ F University, Hangzhou 311300
}

\begin{abstract}
Tree size diversity directly reflects forest ecosystem health and stability. Objective and appropriate evaluation of tree size is essential for understanding the economic, ecological and social value of natural forests or plantations as well as for effective forest stand management. Four distance-free diversity indices (simpson size diversity index, $D_{N}$; shannon size diversity index, $H_{N}$; gini coefficient index of basal area, $G C$; diameter coefficient of variation index, $C V_{d}$ ) and three distance-related indices (simpson size differentiation index, $D_{T}$; shannon size differentiation index, $H_{T}$; mean size differentiation index, $\bar{T}$ ) were selected to analyze the tree size diversity of six types of simulated stands and four measured stand plots with different diameter distribution and spatial patterns. The results show that regardless of extreme that such as compared stands have the same diameter composition but have different size mingling, $G C, C V_{d}, \bar{T}, D_{T}$ and $H_{T}$ can distinguish tree size diversity between stand types which have different diameter distributions objectively and properly. $C V_{d}$ had the best result followed by GC. Accounting for extreme, the distance-related indices, namely $\bar{T}, D_{T}$ and $H_{T}$ can distinguish the difference between different size mingling stands. $C V_{d}$ and $G C$ can be used as the preferred indices in the practical application for calculating simple facilitate. $\bar{T}$ can be used to analyze the dynamic changes of forest structural characteristics for its high distinction ability of spatial
\end{abstract}

收稿日期: 2018-12-26; 接受日期: 2019-03-19

基金项目: 国家自然科学基金(31800539; 31700563; 31870617)、国家林业局林业公益性行业科研专项(20150430303)和宁波市农业科学研究院院长基金 (2018NKYP005)

* 通讯作者 Author for correspondence. E-mail: 506064788@qq.com 
difference that due to its sensitivity to regeneration.

Key words: tree size diversity; diameter distribution; size differentiation; size mingling; size segregation

森林结构的复杂多样通常意味着稳定持久的 生态功能, 提高森林结构多样性可以促进生物多样 性的增加(郑景明和罗菊春, 2003), 近年来的一些研 究甚至认为森林结构多样性对森林生产力或地上 碳储量的影响比生物多样性更大(Ali et al, 2016; Dănescu et al, 2016)。可见对林分结构多样性的研究 具有重要意义。林分结构多样性主要包括林木格局 多样性和林木大小多样性等(Pommerening, 2002)。 其中, 林木大小多样性是描述林分内林木大小的差 异程度, 差异越大的林分空间异质性越强, 能容纳 更多的物种。例如林分径级变异越大, 林分结构越 复杂, 为各种动植物和微生物提供的小生境或食物 的多样性就越高, 林分内总体的生物多样性就越高 (Fries et al, 1997; Rolstad et al, 2001; 雷相东和唐守 正, 2002; 郑景明河罗菊春, 2003)。林木大小多样性 一般采用直径、树高和冠幅 3 个因子来描述, 由于直 径在林分中测定简单、方便、准确(孟宪宇, 2006), 而 且通常与其他因子 (树高和冠幅)之间高度相关 (Lexerød \& Eid, 2006), 因此被普遍用于分析林木大 小多样性(Solomon \& Gove, 1999; Lexerød \& Eid, 2006; McRoberts et al, 2008)。本研究亦采用直径进 行分析。

现代森林生态系统经营策略的基础是提高林 分结构的复杂多样, 以达到可持续经营的目的。为 改善森林经营管理的决策, 描述林木大小多样性的 指数已被引入森林调查和经营研究中, 如何选择新 的或基于现有的知识选择与营林目的相符的、更加 简单适用的大小多样性指数, 对许多森林管理者更 迫切(郑景明和罗菊春, 2003)。目前, 已有描述林木 大小多样性的指数大多与距离无关, 其中, 用径阶 株数或断面积代替树种反映林木大小多样性的 Shannon多样性指数最为常用(雷相东和唐守正, 2002)。此外, 还有分析林木大小不一致性的断面积 Gini系数、直径离散程度的变异系数以及描述直径 分布特征的偏度等(Weiner \& Solbrig, 1984; Neumann \& Starlinger, 2001; Liang et al, 2007; 王懿祥, 2014; Dănescu et al, 2016)。然而, 这些与距离无关 的林木大小多样性指数没有考虑空间结构特征, 不
能全面细微地反映出林分间的差异。事实上, 空间 结构特征决定了林分内的生境和物种多样性, 对林 分的发展有决定性的影响(雷相东和唐守正, 2002)。 Gadow和Füldner (1992)提出的林木大小分化度能度 量出林分内每株林木与其相邻木之间的大小差异 程度, 亦可通过均值反映林分整体的林木大小差异 程度, 易于在森林经营活动中应用。因此, 本研究 分别选用 4 个与距离无关和 3 个与距离有关的林木 大小多样性指数, 结合营林目的比较分析它们表达 林木大小多样性的能力, 为选择合适的森林生物多 样性的结构多样性评价方法和森林经营提供理论 与技术参考。

\section{材料与方法}

\section{1 数据}

\subsection{1 模拟数据}

根据2种林分径级分布类型(倒J型和正态分布) 和3种林分空间分布格局(均匀分布、随机分布和聚 集分布), 分别利用空间分析软件Winkelmass (惠 刚盈等, 2007)模拟6组不同组合的林分类型, 即倒J 均匀、倒J随机、倒J聚集、正态均匀、正态随机、 正态聚集; 每组重复模拟100次。

本研究进一步模拟了极端情况(直径构成相同) 下12块林木大小混交和隔离的林分。其中6块直径 构成相同, 且径级分布呈倒J型: 倒J均匀混交、倒 $\mathrm{J}$ 均匀隔离、倒J随机混交、倒J随机隔离、倒J聚集混 交和倒 J聚集隔离; 另外6块为直径构成相同, 且径 级呈正态分布: 正态均匀混交、正态均匀隔离、正 态随机混交、正态随机隔离、正态聚集混交和正态 聚集隔离(附录1)。

所有模拟林分样地面积均设置为 $50 \mathrm{~m} \times 50 \mathrm{~m}$, 林木株数为 400 株, 林木直径范围 $5-60 \mathrm{~cm}$, 边缘缓 冲区设置为 $5 \mathrm{~m}$ 。

\subsection{2 实测数据}

根据林分直径分布类型和空间分布格局的不 同, 选取 4块实测林分, 分别为正态随机分布的浙 江天目山毛竹(Phyllostachys edulis)纯林、正态均匀 分布的北京中国林业科学研究院院内白皮松(Pinus 
bungeana)人工林、倒J聚集分布的浙江奉化海岛常 绿-落叶阔叶混交林和倒J随机分布的甘肃小陇山百 花林场锐齿栎(Quercus aliena var. acutidentata)落叶 混交林(附录2)。4块实测样地面积均按 $50 \mathrm{~m} \times 50 \mathrm{~m}$ 处理分析，边缘缓冲区设置为 $5 \mathrm{~m}$ 。

\section{2 林木大小多样性指数的评价标准}

林木大小多样性指数是对林木大小差异程度 的量化评价指标, 其评价能力的优劣取决于是否与 营林目的相符, 即与森林经营理念相符。结合 Lexerød和Eid (2006)提出的逻辑排序, 本文得出以 下符合营林目的的逻辑排序: (1)不同径级分布类型 的两个林分, 倒 $\mathrm{J}$ 分布的林分林木大小多样性指数 值应高于正态分布的林分; (2)相同径级分布类型的 两个林分, 林木大小混交状态的林分林木大小多样 性指数值应高于林木大小隔离状态的林分; (3)相同 径级分布类型的两个林分, 径级分布范围广的林分 林木大小多样性指数值应高于分布范围窄的林分; (4)相同径级分布类型的两个林分, 大树株数比例少 但其断面积对总林分断面积影响大的林分，林木大 小多样性指数值应高于大树株数比例少且其断面 积对总林分断面积影响中等的林分, 也高于大树株 数比例多且其断面积总和几乎等于总林分断面积 的林分。此外, 本研究采用林分之间的大小多样性 指数值变异系数 $(C V)$ 评价不同指数的识别能力, $C V$ 值越大说明越能识别不同林分的大小多样性差异 程度, 即指数识别能力越佳。

\section{3 林木大小多样性量化测度指数}

本研究是在林分水平上研究林木大小多样性, 因此选择测度林分水平的 $\alpha$ 多样性指数即应用最广 泛的 Shannon 指数 (Shannon, 1949) 和 Simpson 指数 (Simpson, 1949)。

\subsection{1 与距离无关的林木大小多样性指数}

选择 4 个与距离无关的大小多样性指数, 分别 为Simpson大小多样性指数 $\left(D_{N}\right) 、$ Shannon大小多样 性指数 $\left(H_{N}\right)$ 、断面积Gini系数 $(G C)$ 和直径变异系数 $\left(C V_{d}\right)$, 每个指数的计算方法见表 1 。按 $2 \mathrm{~cm}$ 划分 径级。

\subsection{2 与距离有关的林木大小多样性指数}

选择3个与距离有关的林木大小多样性指数, 分别为Simpson大小分化度指数 $\left(D_{T}\right) 、$ Shannon大小 分化度指数 $\left(H_{T}\right)$ 和大小分化度均值指数 $(\bar{T})$, 每个 指数的计算方法见表 2 。林木大小分化度 $\left(T_{i}\right)$ 是 Gadow和Füldner (1992)研究混交林时提出的概念, 为避免多株最近相邻木的平均值导致潜在折中混 淆的问题，本研究只采用最近一株相邻木进行分 栃 当 $T_{i}=0$ 时说明最近相邻木与参照树的大小相同; $T_{i} \approx 1$ 时则表示最近相邻木与参照树之间相差非 常悬殊。计算 $D_{T}$ 和 $H_{T}$ 时，参考惠刚盈和Gadow (2003) 提出的空间结构参数, 将 $T_{i}$ 划分为 5 个等级: (1) $Q \underline{E}$ $T_{i}<0.2$, 对象木与其最近邻木之间相差 $20 \%$ 以下; (2) $0.2 \leq T_{i}<0.4$, 对象木与其最近邻木之间相差 20\%-40\%; (3) $0.4 \leq T_{i}<0.6$, 对象木与其最近邻木

表 1 与距离无关的林木大小多样性指数

Table 1 Distance-free indices of tree size diversity

\begin{tabular}{llll}
\hline $\begin{array}{l}\text { 指数 } \\
\text { Index }\end{array}$ & $\begin{array}{l}\text { 公式 } \\
\text { Formula }\end{array}$ & $\begin{array}{l}\text { 指数值的理论范围 } \\
\text { Theoretical index value range }\end{array}$ & $\begin{array}{c}\text { 参考文献 } \\
\text { Reference }\end{array}$ \\
\hline Simpson 大小多样性指数 Simpson size diversity index & $D_{N}=1-\sum_{j=1}^{S} p_{j}^{2}$ & {$[0,1]$} & Valbuena, 2012 \\
Shannon 大小多样性指数 Shannon size diversity index & $H_{N}=-\sum_{j=1}^{S} p_{j} \ln \left(p_{j}\right)$ & {$[0, \ln (S)]$} & Buongiorno, 2001 \\
断面积 Gini 系数 Gini coefficient index of basal area & $\sum_{i=1}^{n}(2 i-n-1) B A_{i}$ & {$[0,1]$} & Duduman, 2011 \\
& $G C=\frac{\sum_{i=1}^{n} B A_{i}(n-1)}{n}$ & {$[0,1]$} & Huang, 2000 \\
直径变异系数 Diameter coefficient of variation index & $C V_{d}=\sqrt{\frac{\sum_{i=1}^{n}\left(d_{i}-\bar{d}\right)^{2}}{n-1} / \bar{d}}$ &
\end{tabular}

$S$ 为径阶数, $p_{j}$ 为第 $j$ 径阶林木株数占总株数的频率, $B A_{i}$ 为第 $i$ 级林木断面积 $\left(\mathrm{m}^{2}\right), n$ 为林分内林木总株数, $\bar{d}$ 为林木的平均直径 $(\mathrm{cm})$ 。

$S$ represents number of diameter classes, $p_{j}$ represents proportion of tree numbers in size class $j, B A_{i}$ represents basal area for tree with rank $i\left(\mathrm{~m}^{2}\right), n$ represents total number of trees, $d$ represents mean diameter $(\mathrm{cm})$. 
表 2 与距离有关的林木大小多样性指数

Table 2 Distance-related indices of tree size diversity

\begin{tabular}{|c|c|c|c|}
\hline $\begin{array}{l}\text { 指数 } \\
\text { Index }\end{array}$ & $\begin{array}{l}\text { 公式 } \\
\text { Formula }\end{array}$ & $\begin{array}{l}\text { 指数值理论范围 } \\
\text { Theoretical index value range }\end{array}$ & $\begin{array}{l}\text { 参考文献 } \\
\text { Reference }\end{array}$ \\
\hline Simpson大小分化度指数 Simpson size differentiation index & $D_{T}=1-\sum_{i=1}^{5} P_{i}^{2}$ & {$[0,1]$} & Bai \& Hui, 2016 \\
\hline Shannon大小分化度指数 Shannon size differentiation index & $H_{T}=-\sum_{i=1}^{5} P_{i} \ln \left(P_{i}\right)$ & {$[0, \ln (5)]$} & Bai \& Hui, 2016 \\
\hline 大小分化度均值指数 Mean size differentiation index & $\bar{T}=\frac{1}{N} \sum_{i=1}^{N} T_{i}$ & {$[0,1]$} & Gadow \& Füldner, 1992 \\
\hline & $T_{i}=1-\frac{\min \left(d_{i}, d_{1}\right)}{\max \left(d_{i}, d_{1}\right)}$ & {$[0,1)$} & Gadow \& Füldner, 1992 \\
\hline
\end{tabular}

$P_{i}(i=1, \cdots, 5)$ 为样地核心区内第 $i$ 个大小分化度等级上的林木株数占总株数的频率, $N$ 为核心区内的林木总株数, $T_{i}$ 为第 $i$ 株林木的大小分化 度, $d_{i}$ 为参照木直径, $d_{1}$ 为参照木的最近一株相邻木直径。

$P_{i}(i=1, \cdots, 5)$ represents proportion of tree numbers in size differentiation class $i$ during core area, $N$ represents total number of trees during core area, $T_{i}$ represents size differentiation of tree $i, d_{i}$ represents diameter of reference tree, $d_{1}$ represents diameter of neighborhood tree that nearest reference tree.

之间相差 $40 \%-60 \%$; (4) $0.6 \leq T_{i}<0.8$, 对象木与其 最近邻木之间相差 $60 \%-80 \%$; (5) $0.8 \leq T_{i}<1.0$, 对 象木与其最近邻木之间相差达到 $80 \%$ 及以上。

\section{2 结果}

\section{1 模拟分析}

\subsection{1＼cjkstart不考虑林木的大小隔离和混交情况}

由表 3 和表 4 可知, 除了 $D_{N}$ 和 $H_{N}$, 其他 5 个指数 即 $D_{T} 、 H_{T} 、 \bar{T} 、 G C$ 和 $C V_{d}$ 计算得到的林木大小多样 性指数值均是径级为正态分布的林分低于倒 $\mathrm{J}$ 型的, 7 个指数中只有 $D_{N}$ 和 $H_{N}$ 的评价结果不符合逻辑排 序。从表 3 中指数值的变异系数 $C V$ 可以看出, 符合 逻辑排序的 5 个林木大小多样性指数的识别能力从
大到小依次为 $C V_{d}(42.2 \%)>G C(37.0 \%)>\bar{T}$ $(23.5 \%)>H_{T}(12.1 \%)>D_{T}(7.8 \%)$ 。结合6组模拟林 分指数值之间的两两比较(表4)可以看出, 符合逻辑 排序的 5 个指数即 $D_{T} 、 H_{T} 、 \bar{T} 、 G C$ 和 $C V_{d}$ 均表现为 不同径级分布类型之间的大小多样性指数值具有 显著差异，而相同径级分布类型不同分布格局之间 的大小多样性指数值没有显著差异。可见, 径级分 布类型是影响林木大小多样性的显著因素, 而分布 格局的影响不显著。

在不考虑林木大小是否隔离时，仅从分布格局 和径级分布类型看，只有 5 个林木大小多样性指数, 即 $G C 、 C V_{d} 、 D_{T} 、 H_{T}$ 和 $\bar{T}$ 能客观恰当地表达不同类 型林分的林木大小多样性, 且指数的识别能力从高

表 3 模拟林分的 7 个林木大小多样性指数的范围及变异系数

Table 3 Range and coefficient of variation $(C V)$ of seven tree size diversity indices for the simulated stands

\begin{tabular}{|c|c|c|c|c|c|c|c|}
\hline \multirow{2}{*}{$\begin{array}{l}\text { 指数 } \\
\text { Indices }\end{array}$} & \multicolumn{3}{|c|}{ 正态径级分布 Normal-shaped diameter distribution } & \multicolumn{3}{|c|}{ 倒J径级分布 Inverse J-shaped diameter distribution } & \multirow{2}{*}{$\begin{array}{l}\text { 变异系数 } \\
C V(\%)\end{array}$} \\
\hline & 均匀 Uniform & 随机 Random & 聚集 Aggregation & 均匀 Uniform & 随机 Random & 聚集 Aggregation & \\
\hline$D_{N}$ & $0.930-0.941$ & $0.926-0.942$ & $0.930-0.942$ & $0.814-0.924$ & $0.816-0.913$ & $0.815-0.921$ & 3.5 \\
\hline$H_{N}$ & $2.820-2.964$ & $2.796-2.968$ & $2.801-2.969$ & $1.963-2.790$ & $1.985-2.724$ & $2.011-2.783$ & 10.1 \\
\hline$G C$ & $0.269-0.317$ & $0.270-0.323$ & $0.271-0.313$ & $0.520-0.627$ & $0.530-0.631$ & $0.529-0.639$ & 37.0 \\
\hline$C V_{d}$ & $0.259-0.305$ & $0.255-0.308$ & $0.253-0.298$ & $0.532-0.684$ & $0.547-0.691$ & $0.543-0.704$ & 42.2 \\
\hline$D_{T}$ & $0.617-0.696$ & $0.624-0.696$ & $0.616-0.694$ & $0.727-0.773$ & $0.731-0.775$ & $0.733-0.779$ & 7.8 \\
\hline$H_{T}$ & $1.070-1.267$ & $1.092-1.279$ & $1.049-1.274$ & $1.361-1.524$ & $1.366-1.533$ & $1.373-1.548$ & 12.1 \\
\hline $\bar{T}$ & $0.238-0.303$ & $0.234-0.297$ & $0.227-0.298$ & $0.334-0.464$ & $0.345-0.452$ & $0.351-0.464$ & 23.5 \\
\hline
\end{tabular}

$D_{N}, H_{N}, G C, C V_{d}, D_{T}, H_{T}$ 和 $\bar{T}$ 见表1、2。

$D_{N}, H_{N}, G C, C V_{d}, D_{T}, H_{T}$ and $\bar{T}$ as in Table 1 and 2. 
到低依次为 $C V_{d}>G C>\bar{T}>H_{T}>D_{T}$ 。

\subsection{2 考虑林木的大小隔离和混交情况}

从表 5 可以看出, 对于直径构成相同但大小混 交程度不同的林分, 4 个与距离无关的林木大小多 样性指数, 即 $D_{N} 、 H_{N} 、 G C$ 和 $C V_{d}$ 的计算值是完全相 同的，显然，这 4 个指数无法识别出这些林分之间 大小混交差别的细微空间特征。

从表 5 可以看出, 与距离有关的林木大小多样 性指数 $\bar{T} 、 D_{T}$ 和 $H_{T}$ 能很好地识别直径构成相同但大 小混交程度不同的空间特征差异, 都表现为林木大 小隔离的林分指数值远远低于大小混交的林分。值 得注意的是, 倒 $\mathrm{J}$ 径级分布且大小隔离林分的林木 大小多样性指数值远远低于正态径级分布且大小 混交的林分。由此可见, 林木大小是否隔离应该作
为选择林木大小多样性指数时首要考虑的因素。在 林木大小混交时, 3 个与距离有关的林木大小多样 性指数都表现为径级分布为倒 $\mathrm{J}$ 型的林分林木大小 多样性高于正态分布的林分; 在林木大小隔离时 (表5), 只有 $\bar{T}$ 的指数值表现为倒 $\mathrm{J}$ 型的略高于正态 分布的林分。

综上所述，与距离无关的指数 $D_{N}$ 和 $H_{N}$ 不能符 合逻辑地识别不同径级分布林分的大小多样性; 符 合逻辑排序的 $G C$ 和 $C V_{d}$ 不能识别直径构成相同但 大小混交程度不同林分的大小多样性差异。与距离 有关的 $D_{T}$ 和 $H_{T}$ 只能在林木大小混交情况下符合逻 辑排序地识别不同径级分布林分的大小多样性，而 $\bar{T}$ 则能在林木大小混交或隔离情况下较好识别出 不同径级分布类型林分的林木大小多样性。

表 4 模拟林分的 7 个林木大小多样性指数均值(标准差)

Table 4 Mean (standard deviation) of seven tree size diversity indices for the simulated stands

\begin{tabular}{|c|c|c|c|c|c|c|}
\hline \multirow{2}{*}{$\begin{array}{l}\text { 指数 } \\
\text { Indices }\end{array}$} & \multicolumn{3}{|c|}{ 正态径级分布 Normal-shaped diameter distribution } & \multicolumn{3}{|c|}{ 倒J径级分布 Inverse J-shaped diameter distribution } \\
\hline & 均匀 Uniform & 随机 Random & 聚集 Aggregation & 均匀 Uniform & 随机 Random & 聚集 Aggregation \\
\hline$D_{N}$ & $0.936(0.002)^{\mathrm{a}}$ & $0.936(0.003)^{\mathrm{a}}$ & $0.935(0.002)^{\mathrm{a}}$ & $0.877(0.022)^{b}$ & $0.877(0.022)^{\mathrm{b}}$ & $0.881(0.023)^{b}$ \\
\hline$H_{N}$ & $2.890(0.033)^{\mathrm{a}}$ & $2.892(0.039)^{\mathrm{a}}$ & $2.884(0.032)^{\mathrm{a}}$ & $2.394(0.169)^{\mathrm{bc}}$ & $2.388(0.166)^{\mathrm{c}}$ & $2.426(0.176)^{\mathrm{b}}$ \\
\hline GC & $0.292(0.010)^{\mathrm{a}}$ & $0.291(0.011)^{\mathrm{a}}$ & $0.290(0.010)^{\mathrm{a}}$ & $0.588(0.023)^{b}$ & $0.585(0.022)^{\mathrm{b}}$ & $0.589(0.023)^{\mathrm{b}}$ \\
\hline$C V_{d}$ & $0.278(0.010)^{\mathrm{a}}$ & $0.278(0.011)^{\mathrm{a}}$ & $0.276(0.010)^{\mathrm{a}}$ & $0.625(0.034)^{b}$ & $0.621(0.032)^{\mathrm{b}}$ & $0.627(0.033)^{\mathrm{b}}$ \\
\hline$D_{T}$ & $0.657(0.016)^{\mathrm{a}}$ & $0.659(0.017)^{\mathrm{a}}$ & $0.656(0.016)^{\mathrm{a}}$ & $0.757(0.010)^{b}$ & $0.757(0.009)^{\mathrm{b}}$ & $0.759(0.010)^{\mathrm{b}}$ \\
\hline$H_{T}$ & $1.173(0.041)^{\mathrm{a}}$ & $1.180(0.043)^{\mathrm{a}}$ & $1.171(0.042)^{\mathrm{a}}$ & $1.463(0.037)^{\mathrm{b}}$ & $1.462(0.035)^{\mathrm{b}}$ & $1.470(0.038)^{\mathrm{b}}$ \\
\hline $\bar{T}$ & $0.262(0.013)^{\mathrm{a}}$ & $0.262(0.014)^{\mathrm{a}}$ & $0.261(0.013)^{\mathrm{a}}$ & $0.404(0.025)^{b}$ & $0.403(0.025)^{\mathrm{b}}$ & $0.406(0.026)^{\mathrm{b}}$ \\
\hline
\end{tabular}

$D_{N}, H_{N}, G C, C V_{d}, D_{T}, H_{T}$ 和 $\bar{T}$ 见表1、2。同一行不同小写字母表示差异显著(LSD检验, $P<0.05$ )。

$D_{N}, H_{N}, G C, C V_{d}, D_{T}, H_{T}$ and $\bar{T}$ as in Table 1 and 2. Different letters indicate significant differences in the same row (LSD test, $P<0.05$ ).

表 512 块不同林木大小混交与大小隔离的模拟林分林木大小多样性指数值

Table 5 Tree size diversity indices of different size mingling and size segregation for twelve different simulated stands

\begin{tabular}{llllllll}
\hline 林分类型 Stand type & $D_{N}$ & $H_{N}$ & $G C$ & $C V_{d}$ & $D_{T}$ & $H_{T}$ & $\bar{T}$ \\
\hline 倒J均匀混交 Inverse J-shaped uniform mingling type & 0.897 & 2.610 & 0.633 & 0.692 & 0.770 & 1.512 & 0.437 \\
倒J均匀隔离 Inverse J-shaped uniform segregation type & 0.897 & 2.610 & 0.633 & 0.692 & 0.054 & 0.154 & 0.067 \\
倒J随机混交 Inverse J-shaped random mingling type & 0.897 & 2.610 & 0.633 & 0.692 & 0.769 & 1.510 & 0.421 \\
倒J随机隔离 Inverse J-shaped random segregation type & 0.897 & 2.610 & 0.633 & 0.692 & 0.049 & 0.140 & 0.050 \\
倒J聚集混交 Inverse J-shaped aggregated mingling type & 0.897 & 2.610 & 0.633 & 0.692 & 0.772 & 1.521 & 0.421 \\
倒J聚集隔离 Inverse J-shaped aggregated segregation type & 0.897 & 2.610 & 0.633 & 0.692 & 0.035 & 0.107 & 0.041 \\
正态均匀混交 Normal-shaped uniform mingling type & 0.937 & 2.906 & 0.299 & 0.282 & 0.673 & 1.208 & 0.275 \\
正态均匀隔离 Normal-shaped uniform segregation type & 0.937 & 2.906 & 0.299 & 0.282 & 0.078 & 0.196 & 0.040 \\
正态随机混交 Normal-shaped random mingling type & 0.937 & 2.906 & 0.299 & 0.282 & 0.665 & 1.184 & 0.269 \\
正态随机隔离 Normal-shaped random segregation type & 0.937 & 2.906 & 0.299 & 0.282 & 0.044 & 0.123 & 0.032 \\
正态聚集混交 Normal-shaped aggregated mingling type & 0.937 & 2.906 & 0.299 & 0.282 & 0.660 & 1.177 & 0.268 \\
正态聚集隔离 Normal-shaped aggregated segregation type & 0.937 & 2.906 & 0.299 & 0.282 & 0.030 & 0.085 & 0.024 \\
\hline
\end{tabular}

$D_{N}, H_{N}, G C, C V_{d}, D_{T}, H_{T}$ 和 $\bar{T}$ 见表1、2。

$D_{N}, H_{N}, G C, C V_{d}, D_{T}, H_{T}$ and $\bar{T}$ as in Table 1 and 2. 
表 64 块实测样地的林木大小多样性指数值

Table 6 Tree size diversity indices for four measured plots

\begin{tabular}{llllll}
\hline 指数 Index & $\mathrm{HZ}$ & $\mathrm{NB}$ & $\mathrm{BJ}$ & $\mathrm{GS}$ & $\mathrm{CV}(\%)$ \\
\hline$G C$ & 0.16 & 0.543 & 0.204 & 0.553 & 0.581 \\
$C V_{d}$ & 0.146 & 0.554 & 0.186 & 0.566 & 0.628 \\
$D_{T}$ & 0.330 & 0.726 & 0.489 & 0.750 & 0.350 \\
$H_{T}$ & 0.557 & 1.368 & 0.792 & 1.421 & 0.413 \\
$\bar{T}$ & 0.126 & 0.324 & 0.169 & 0.396 & 0.502
\end{tabular}

HZ: 浙江毛竹林; NB: 浙江混交林; BJ: 北京白皮松林; GS: 甘肃混交 林。CV: 变异系数。 $D_{N}, H_{N}, G C, C V_{d}, D_{T}, H_{T}$ 和 $\bar{T}$ 见表 $1 、 2 。$

HZ, Phyllostachys edulis forest in Zhejiang Province; NB, Mixed forest in Zhejiang Province; BJ, Pinus Bungeana plantation in Beijing; GS, Mixed forest in Gansu Province. $C V$, Coefficient of variation. $D_{N}, H_{N}, G C, C V_{d}$, $D_{T}, H_{T}$ and $\bar{T}$ as in Table 1 and 2 .

\section{2 实例分析}

采用 $G C 、 C V_{d} 、 D_{T} 、 H_{T}$ 和 $\bar{T}$ 这 5 个指数进行实 例分析。从表 6 可以看出, 5 个林木大小多样性指数 值均呈现为甘肃混交林(GS) > 浙江混交林(NB) > 北京白皮松林(BJ) > 浙江毛竹林(HZ), 都能符合逻 辑排序地表达出径级分布呈倒J型的2块林分(NB和 GS)的林木大小多样性指数值高于呈正态分布的 2 块林分(BJ和HZ)。从2块径级分布呈倒J型的林分看, GS的林木径阶范围在 $[6 \mathrm{~cm}, 46 \mathrm{~cm}], \mathrm{NB}$ 的林木径阶 范围在 $[6 \mathrm{~cm}, 42 \mathrm{~cm}]$, 这2块林分的径级分布范围相 似, 从附录 2 可以看出 GS 和NB的大树比例少, 但 GS中的大树断面积对林分总断面积的贡献高于NB, 这可能是GS的指数值高于NB的缘由。BJ和HZ的径 级都呈正态分布, BJ林龄较HZ成熟得多, 由于年龄 上的差异, BJ $[10 \mathrm{~cm}, 38 \mathrm{~cm}]$ 的径级分布范围比 $\mathrm{HZ}$ $[6 \mathrm{~cm}, 16 \mathrm{~cm}]$ 的大得多, 因此, BJ林分的林木大小多 样指数值远远高于 $\mathrm{HZ}$ 。从各实测样地指数值的变异 系数 $C V$ 来看, 各指数的识别能力从大到小排序仍 然为 $C V_{d}>G C>\bar{T}>H_{T}>D_{T}$ 。

\section{3 讨论}

本文基于白超(2016)的研究，结合营林目的， 通过模拟数据进一步分析 7 个林木大小多样性指数 的表达能力。结果表明, 在林分水平上分布格局对 林木大小多样性无显著影响, 但径级分布类型对林 木大小多样性有显著影响。值得注意的是, 在林木 大小隔离时, 径级分布为倒 $\mathrm{J}$ 型的林分林木大小多 样性指数可能低于正态分布的林分。不过在现实林 分中, 林分径级分布呈倒 $\mathrm{J}$ 型且大小隔离的情况较 为少见, 可能出现于由多个不同林龄组的同龄林构 成的人工林中。林分的径级变异程度是诸多因素共
同作用的结果，这些因素来源可分为外部与内部两 类, 如水分、土壤、温度和小地形等为外部因素, 内 部因素如树种、年龄和更新等。目前，一般认为天 然林的径级分布呈倒J型，其林木大小多样性高于 呈正态分布的人工林(Spies，1998; Lexerød \& Eid, 2006; O’hara et al, 2007)。由于不同种群的内禀增长 率不同，不同植物种群的个体大小差异大，树种丰 富的天然林往往能形成较为复杂多样的林分结构。 “生态优先”的林业经营理念以森林可持续经营为基 础(寇文正等, 2000), 而林下更新是实现森林可持续 经营的关键(黄清麟, 2005)。从人工林的正态径级分 布可知小径级林木少，意味着林下更新差; 而呈倒 $\mathrm{J}$ 型径级分布的天然林具有众多的小径级林木，林下 更新丰富, 充足的林下更新为径级变异提供动力来 源, 提高林木大小多样性的同时促进林分结构的空 间异质性。从空间分布格局上看，种子在母树周边 天然更新，从而形成不同大小的林木混交的情况， 也就是说当林下幼树更新好则会呈现出林木大小 混交的格局，进而提高林分的林木大小多样性。 Wang等(2011)和Lei等(2009)的研究认为择伐或部分 采伐通过改善林内环境促进林下幼树更新，进而提 高林木大小多样性。Varga (2005)和Lei等(2009)的研 究认为林分成熟度促进林分的大小多样性, 随着林 龄的增长, 径级结构的变异加大, 林分拥有更广的 径级分布范围和更多的大径级林木，直接构成林分 垂直和水平结构上的复杂多样性，进而提高林分的 林木大小多样性。

本研究认为不用主观划分等级的 $C V_{d} 、 G C$ 和 $\bar{T}$ 能较好识别林分的林木大小多样性差异。Lexerød 和Eid (2006)与Valbuena (2012)的研究也指出, 避免 主观分组的林木大小多样性指数因为尽可能地保 留了原数据包含的所有信息，所以具有更好的表达 能力。到目前为止, 以径阶株数或断面积代替树种 反映林木大小多样性的指数应用最广, 以断面积计 算的Shannon大小多样性指数(Schulte \& Buongiorno, 1998; Wikström \& Eriksson, 2000; Buongiorno, 2001; Staudhammer \& Lemay, 2001; 舒树沝, 2015)比较常 见，例如向玮等(2011)以Shannon断面积多样性指数 引入矩阵生长模型; 以株数计算的林木大小多样性 (李超, 2016, 2017; 常荣涛, 2014; 赵中华等, 2019) 也不少，例如常荣涛(2014)采用Shannon直径(株数) 大小多样性和物种多样性的二维联合熵分析栎类 
和阔叶混交林林分结构多样性的动态。虽然 Shannon大小多样性指数应用最广, 但本研究与 Rouvinen和Kuuluvainen (2005)的结果都认为以株 数计算的Shannon大小多样性指数不能恰当识别不 同类型林分的林木大小多样性。不过以断面积计算 的Shannon大小多样性指数表达能力如何, 还有待 进一步分析研究。

本研究仅从林分直径分布类型和林木分布格 局模拟分析评价了 7 个林木大小多样性指数的表达 能力，尚有待于进一步系统分析评价林木径级分布 范围和大树断面积占总林分断面积比例不同等情 况下不同大小多样性指数的表达能力。

\section{参考文献}

Ali A, Yan ER, Chen HYH, Chang SX, Zhao YT, Yang XD, Xu MS (2016) Stand structural diversity rather than species diversity enhances aboveground carbon storage in secondary subtropical forests in Eastern China. Biogeosciences, 13, 4627-4635.

Bai C, Hui GY (2016) Study on diversity indices of tree diameter size. Forest Research, 29, 340-347. (in Chinese with English abstract) [白超, 惠刚盈 (2016) 林木直径大 小多样性量化测度指数的比较研究. 林业科学研究, 29, 340-347.]

Buongiorno J (2001) Quantifying the implications of transformation from even to uneven-aged forest stands. Forest Ecology and Management, 151, 121-132.

Chang RT (2014) Dynamic research on stand structure diversity of nature forest in short time. Journal of Henan Forestry Science and Technology, 34(2), 8-12. (in Chinese with English abstract) [常荣涛 (2014) 栎类和阔叶混交林林分 结构多样性的动态研究. 河南林业科技, 34(2), 8-12.]

Dănescu A, Albrecht AT, Bauhus J (2016) Structural diversity promotes productivity of mixed, uneven-aged forests in southwestern Germany. Oecologia, 182, 319-333.

Duduman GA (2011) Forest management planning tool to create highly diverse uneven-aged stands. Forestry, 84, 301-314.

Fries C, Johansson O, Pettersson B, Simonsson P (1997) Silvicultural models to maintain and restore natural stand structures in Swedish boreal forests. Forest Ecology and Management, 94, 89-103.

Gadow K, Füldner K (1993) Zur Methoik der Bestandesbeschreibung. Vortrag anlaesslich der Jahrestagung der A G Forsteinrichtung in Kliekenb. Dessau. (in German)

Huang LW (2000) Principles of Statistics. China Statistics Press, Beijing. (in Chinese) [黄良文 (2000) 统计学原理. 中国统计出版社, 北京.]

Huang QL (2005) Preliminary introduction to "close to nature forest management" in Germany. World Forestry Research, 18(3), 73-77. (in Chinese with English abstract) [黄清麟 (2005) 浅谈德国的“近自然森林经营”. 世界林业研究,
18(3), 73-77.]

Hui GY, Li L, Zhao ZH, Dang PX (2007) The comparison of methods in analysis of the tree spatial distribution pattern. Acta Ecological Sinica, 27, 4717-4728. (in Chinese with English abstract) [惠刚盈，李丽，赵中华，党普兴 (2007) 林木空间分布格局分析方法. 生态学报, 27, 4717-4728.]

Hui GY, Pommerening A (2014) Analysing tree species and size diversity patterns in multi-species uneven-aged forests of Northern China. Forest Ecology and Management, 316, 125-138.

Hui GY, Gadow VK (2003) Quantitative Analysis of Forest Spatial Structure. China Science and Technology Press, Beijing. (in Chinese) [惠刚盈, Gadow VK (2003) 森林空 间结构量化分析方法. 中国科学技术出版社, 北京.]

Kou WZ, Yang JZ, Yuan SQ (2000) Preliminary discussion on forestry management ideas of "Eco-Priority". Forestry Economics, (1), 1-6. (in Chinese) [寇文正, 杨建洲, 袁少青 (2000) 试论“生态优先”的林业经营思想. 林业经济, (1), 1-6.]

Lei XD, Tang SZ (2002) Indicators on structural diversity within stand: A review. Scientia Silvae Sinicae, 38(3), 140-146. (in Chinese with English abstract) [雷相东, 唐守 正 (2002) 林分结构多样性指标研究综述. 林业科学, 38(3), 140-146.]

Lei XD, Wang W, Peng CH (2009) Relationships between stand growth and structural diversity in spruce-dominated forests in New Brunswick, Canada. Canadian Journal of Forest Research, 39, 1835-1847.

Lexerød NL, Eid T (2006) An evaluation of different diameter diversity indices based on criteria related to forest management planning. Forest Ecology and Management, 222, 17-28.

Li C, Lü YY, Xu H, Wei AC, Xiong HX, Zhang B, Sun XL, Xu TT, Shi XL, Ou GL (2017) Stand diameter size diversity in Pinus kesiya var. langbianensis natural forests. Journal of Yunnan Agricultural University (Natural Science), 32, 1108-1120. (in Chinese with English abstract) [李超, 问妍 宇, 胥辉, 魏安超, 熊河先, 张博, 孙雪莲, 徐婷婷, 石晓 琳, 欧光龙 (2017) 思茅松天然林林分直径大小多样性 研究. 云南农业大学学报(自然科学), 32, 1108-1120.]

Li C, Lü YY, Xu H, Xu TT, Zhang B, Wei AC, Sun XL, Xiong HX, Shi XL, Ou GL (2016) Stand diameter size diversity and their environmental explanation in Pinus kesiya var. langbianensis natural forests. Journal of Northeast Forestry University, 44(11), 24-30. (in Chinese with English abstract) [李超, 问妍宇, 胥辉, 徐婷婷, 张博, 魏安超, 孙雪莲, 熊河先, 石晓琳, 欧光龙 (2016) 思茅松天然林 林分直径大小多样性及环境解释. 东北林业大学学报, 44(11), 24-30.]

Liang J, Buongiorno J, Monserud RA, Kruger EL, Zhou M (2007) Effects of diversity of tree species and size on forest basal area growth recruitment and mortality. Forest Ecology and Management, 243, 116-127.

McRoberts RE, Winter S, Chirici G, Hauk E, Pelz DR, Moser WK, Hatfield MA (2008) Large-scale spatial patterns of forest structural diversity. Canadian Journal of Forest 
Research, 38, 429-438.

Meng XY (2006) Forest Mensuration, 3rd edn. (in Chinese). China Forestry Publishing House, Beijing. (in Chinese) [孟 宪宇 (2006) 测树学 (第3版). 中国林业出版社, 北京.]

Neumann M, Starlinger F (2001) The significance of different indices for stand structure and diversity in forests. Forest Ecology and Management, 145, 91-106.

O’hara KL, Hasenauer H, Kindermann G (2007) Sustainability in multi-aged stands: An analysis of long-term plenter systems. Forestry, 80, 163-181.

Pommerening A (2002) Approaches to quantifying forest structures. Forestry, 75, 305-324.

Rolstad J, Gjerde I, Olaf SK, Rolstad E (2001) Epiphytic lichens in Norwegian coastal spruce forest, historic logging and present forest structure. Ecological Applications, 11, 421-436.

Rouvinen S, Kuuluvainen T (2005) Tree diameter distributions in natural and managed old Pinus sylvestris-dominated forests. Forest Ecology and Management, 208, 45-61.

Schulte BJ, Buongiorno J (1998) Effects of uneven-aged silviculture on the stand structure, species composition, and economic returns of loblolly pine stands. Forest Ecology and Management, 111, 83-101.

Shannon CE (1949) Communication theory of secrecy systems. The Bell System Technical Journal, 28, 656-715. factors of forest diversity in Yunnan pine secondary forest based on structural equation model. Journal of Northeast Forestry University, 43(10), 63-67. (in Chinese with English abstract) [舒树丞, 赵洋毅, 段旭, 胡慧蓉, 熊好琴 (2015) 基于结构方程模型的云南松次生林林木多样性影响因子. 东北林业大学学报, 43(10), 63-67.]

Simpson EH (1949) Measurement of diversity. Nature, 163, 688.

Solomon DS, Gove JH (1999) Effects of uneven-age management intensity on structural diversity in two major forest types in New England. Forest Ecology and Management, 114, 265-274.

Spies TA (1998) Forest structure: A key to the ecosystem. Northwest Science, 72, 34-36.

Staudhammer CL, Lemay VM (2001) Introduction and evaluation of possible indices of stand structural diversity. Canadian Journal of Forest Research, 31, 1105-1115.

Valbuena R, Packalén P, Marti S, Maltamo M (2012) Diversity

\section{附录 Supplementary Material}

附录1 12块林木大小混交与大小隔离的模拟林分格局分布图

Appendix 1 Interspersion of tree of different size mingling and size segregation for 12 simulated stands http://www.biodiversity-science.net/fileup/PDF/2018341-1.pdf

附录2 4块实测样地径级分布直方图

Appendix 2 Histograms of diameter distribution for four measured plots http://www.biodiversity-science.net/fileup/PDF/2018341-2.pdf 


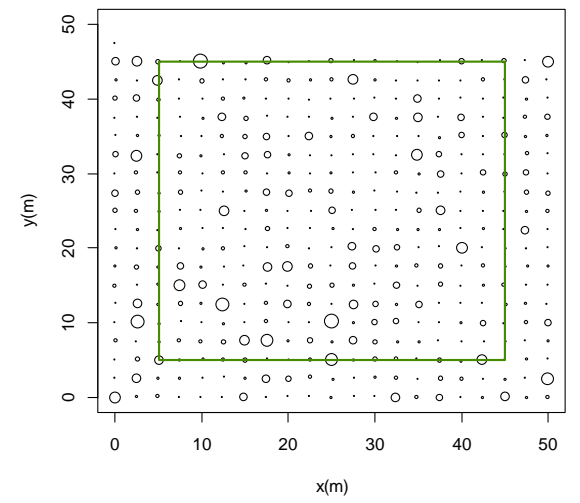

倒 J 均匀混交

Inverse J-shaped uniform mingling type

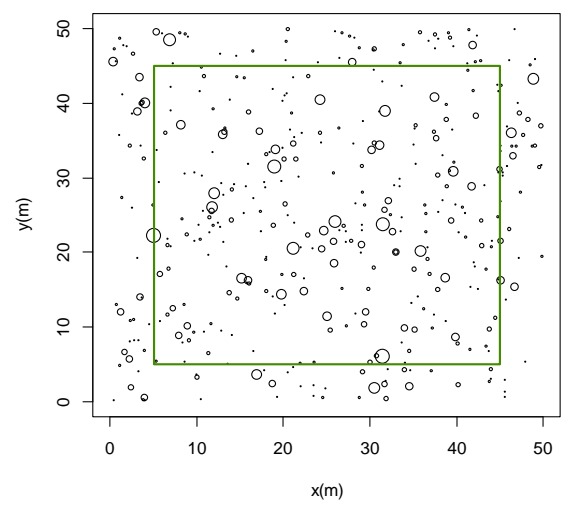

倒 J 随机混交

Inverse J-shaped random mingling type

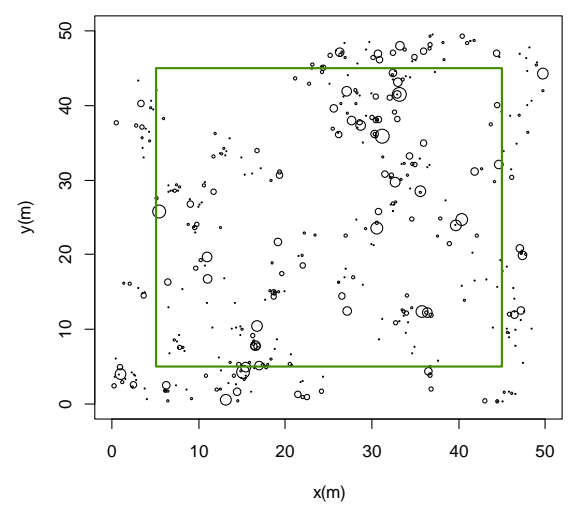

倒 J 聚集混交

Inverse J-shaped aggregated mingling type

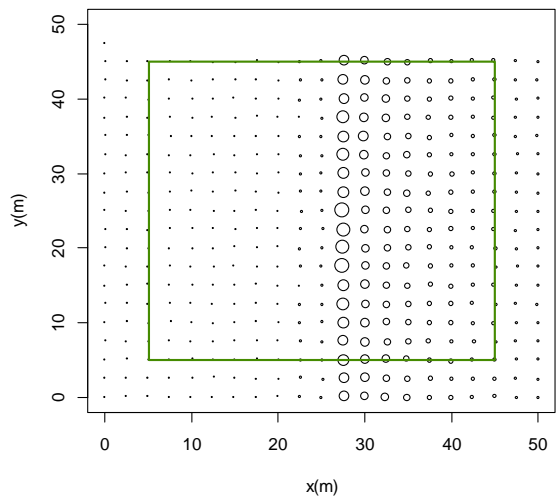

倒 J 均匀隔离

Inverse J-shaped uniform segregation type

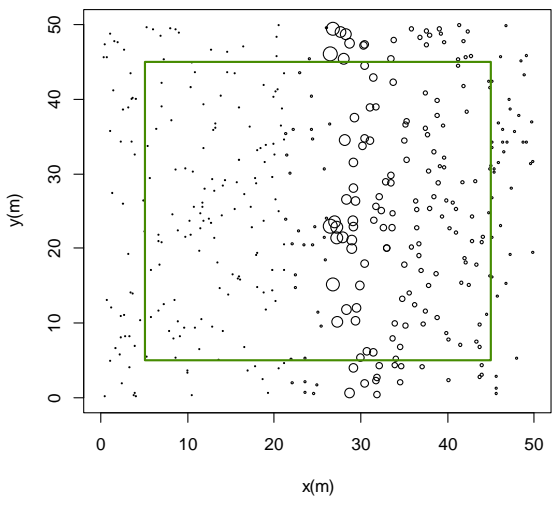

倒 J 随机隔离

Inverse J-shaped random segregation type

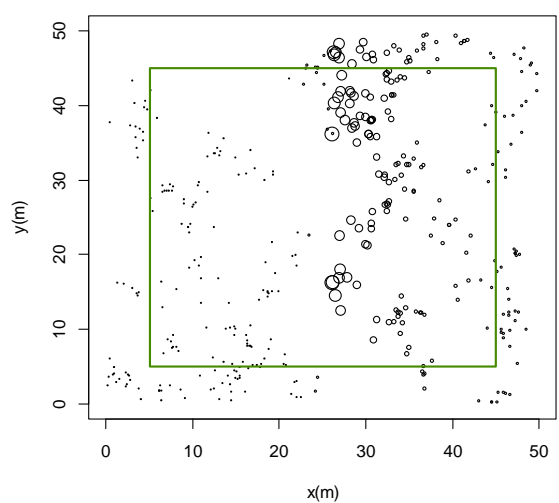

倒 J 聚集隔离

Inverse J-shaped aggregated segregation type 


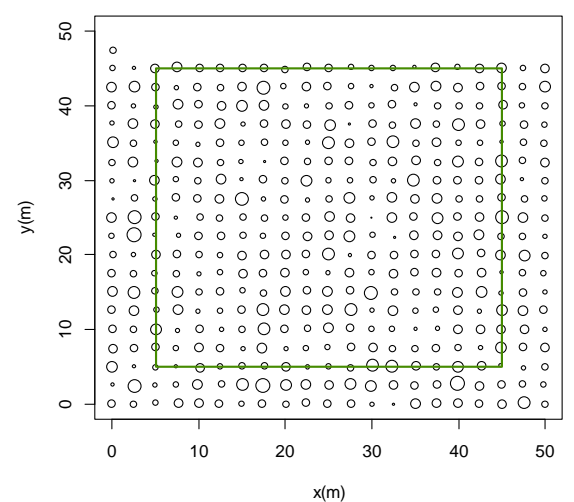

正态均匀混交

Normal-shaped uniform mingling type

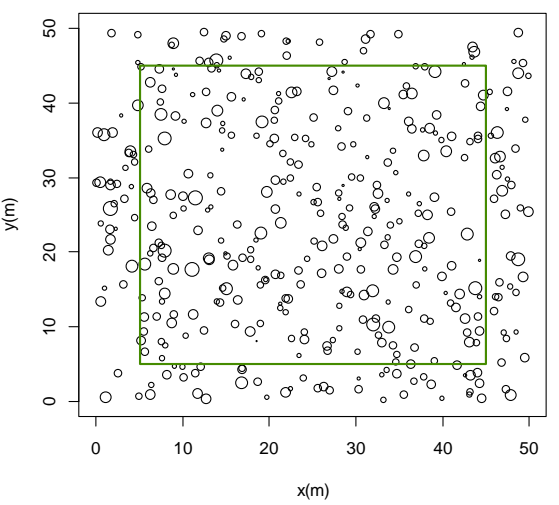

正态随机混交

Normal-shaped random mingling type

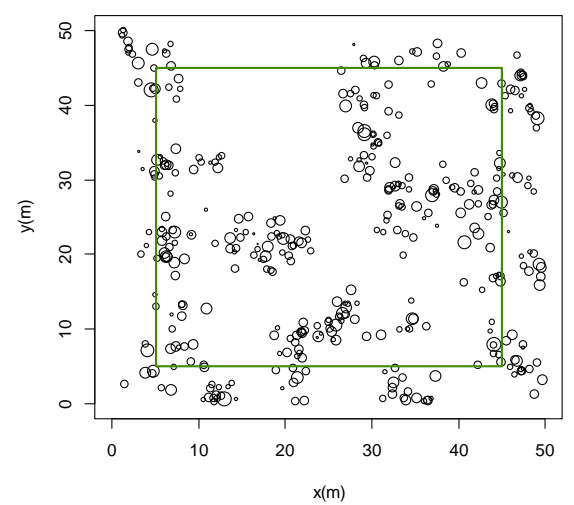

正态聚集混交

Normal-shaped aggregated mingling type

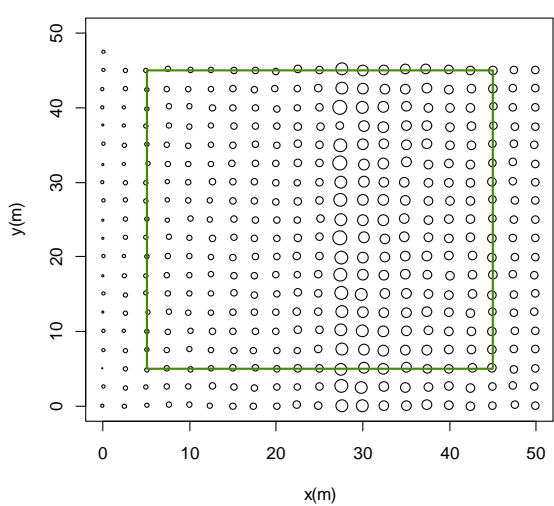

正态均匀隔离

Normal-shaped uniform segregation type

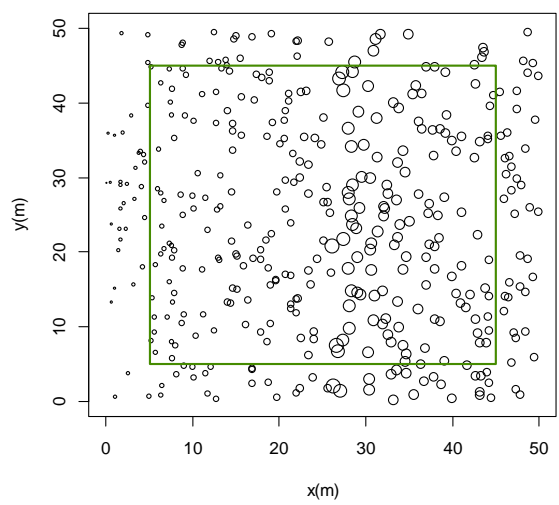

正态随机隔离

Normal-shaped random segregation type

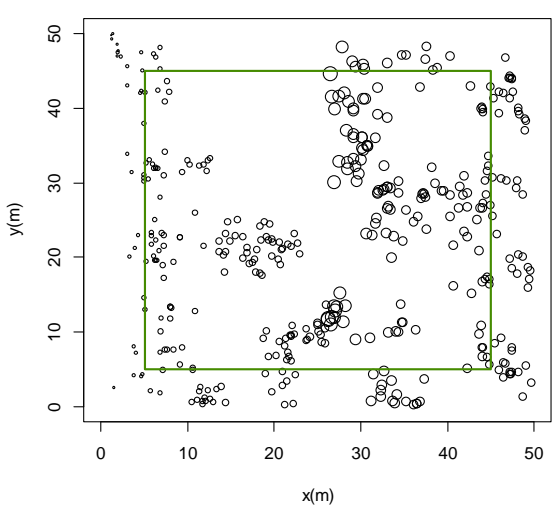

正态聚集隔离

Normal-shaped aggregated segregation type
2

附录 112 块林木大小混交与大小隔离的模拟林分格局分布图

4 


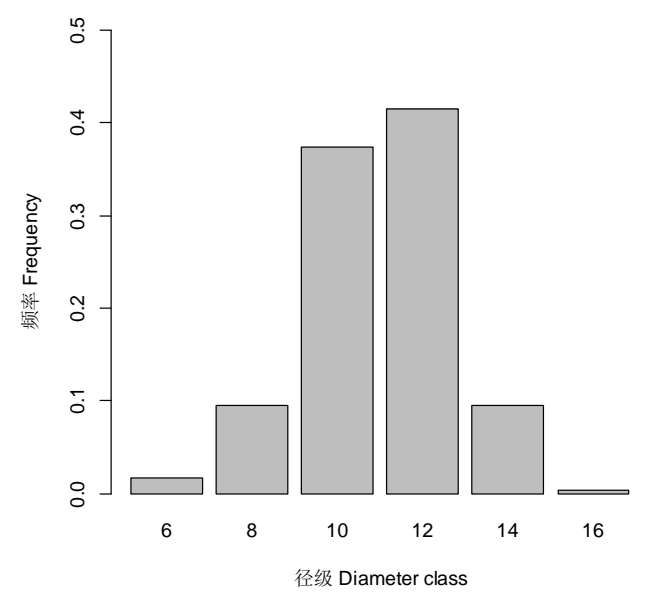

C

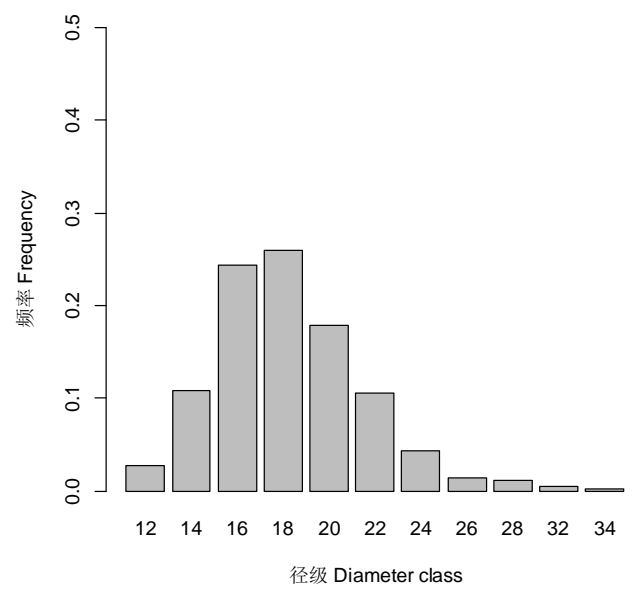

B

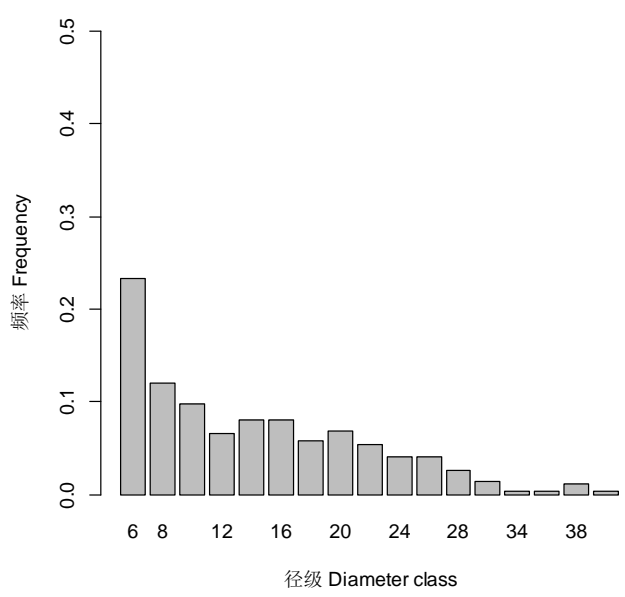

D

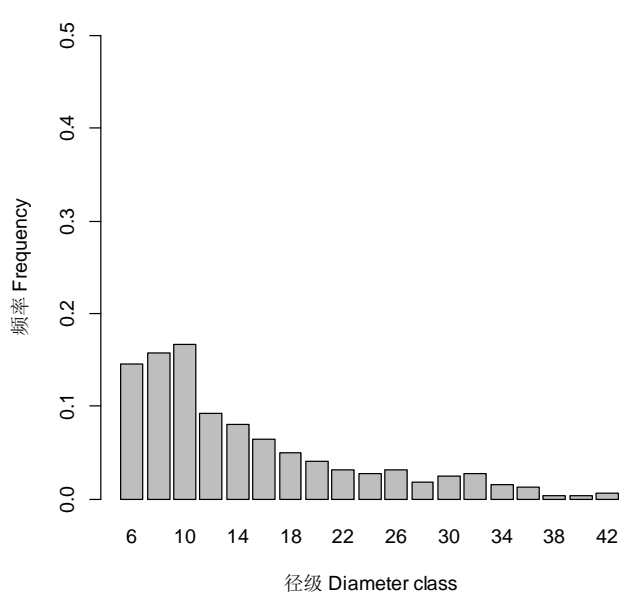

附录 24 块实测样地径级分布直方图。(A)浙江毛竹林, 共 1,489 株林木, 呈正态随机分布 $(\bar{W}=0.515)$; (B)浙江混交林, 共 275 株林木, 呈倒 $\mathbf{J}$ 聚集分布 $(\bar{W}=\mathbf{0 . 5 3 6})$; (C)北京白皮松林，共 369 株林木，呈正态均 匀分布 $(\bar{W}=0.421)$; (D)甘肃混交林, 共 323 株林木, 呈倒 $\mathbf{J}$ 随机分布 $(\bar{W}=0.506)$ 。林木直径划分等级为 2 $\mathrm{cm} ; \bar{W}$ 为角尺度均值, 当 $\bar{W}<0.475$ 时为均匀分布, $0.475 \leq \bar{W} \leq 0.517$ 时为随机分布, 当 $\bar{W}>0.517$ 时为聚 集分布。

Appendix 2 Histograms of diameter distribution for four measured plots. (A) P. edulis forest in Zhejiang Province, normal-shaped random distribution $(\bar{W}=0.515)$ and have 1,489 trees; (B) Mixed forest in Zhejiang Province, inverse J-shaped aggregated distribution $(\bar{W}=0.536)$ and have 275 trees; $(C)$ P. bungeana plantation in Beijing, normal-shaped uniform distribution ( $\bar{W}=0.421)$ and have 369 trees; (D) Mixed forest in Gansu Province, inverse J-shaped random distribution $(\bar{W}=0.506)$ and have 323 trees. Class of diameter is $2 \mathrm{~cm}, \bar{W}$ represents mean uniform angle index, uniform distribution for $\bar{W}<0.475$; random distribution for $0.475 \leq \bar{W} \leq 0.517$; aggregated distribution for $\bar{W}>0.517$. 